\title{
Otimização multicritério da extração madeireira na Amazônia com o uso de escaneamento laser aerotransportado
}

\author{
Multi-criteria optimization for log extraction in \\ Amazon based on airborne laser scanning data
}

\author{
Rauff Pereira Barbosa1, Luiz Carlos Estraviz Rodriguez² e Eric Bastos Gorgens ${ }^{3}$
}

\begin{abstract}
Resumo
Diversos fatores, muitas vezes antagônicos, influenciam a definição dos ramais de extração de madeira na Amazônia. A falta de dados detalhados para planejamento pode tornar o plano de exploração precário, causando danos ambientais e altos custos de implantação e manutenção das trilhas de arraste. O objetivo deste estudo foi utilizar a otimização multicritério para determinar a rota ótima de extração madeireira a partir de informações derivadas do levantamento laser aerotransportado combinadas em quatro diferentes composições de custo. O cenário de minimização da distância resultou em 26,95 km de trilhas e 30 interceptações de Áreas de Preservação Permanente (APP). Por sua vez, o cenário de minimização do impacto à vegetação resultou $26,15 \mathrm{~km}$ de trilhas e 42 interceptações de APP. O cenário de minimização de rampas resultou em 30,92 km de trilhas e 52 interceptações de APP. Por fim, o cenário de minimização de impacto na APP apresentou $26,31 \mathrm{~km}$ de trilhas e 17 interceptações. Os resultados indicam que os cenários mais adequados são: minimização de impacto à APP e minimização balanceada.
\end{abstract}

Palavras-chave: colheita florestal, LiDAR, manejo de precisão.

\begin{abstract}
Several factors affect the management of natural forests, which many times are antagonistic, when dealing with the establishment and location of skid trails. The lack of detailed data about the forest structure and topography make the decision making process precarious. The use of airborne laser scanning may improve the capacity to obtain adequate information about remote areas with dense vegetation. This study suggests using the multi criteria optimization methods to determine the optimal harvesting path subject to four different cost scenarios. The optimization process with equal weights resulted in a total travelled distance equals to 26.95 kilometers with the permanent preservation area crossed 30 times. The minimization of impacts scenario resulted on a total travelled distance equals to $26.15 \mathrm{~km}$ with the preserved area traversed across 42 times, and the overall ramp slope minimization resulted in a total travelled distance of $30.92 \mathrm{~km}$ with 52 crossings of the preserved area. Finally, the minimization of crossings in the permanently preserved area resulted on a total travelled distance equals to $26.15 \mathrm{~km}$ with 17 interceptions. The objective of exploring a set of logging strategies with multi-criteria optimization methods was reached. Based on the results, the best scenarios were minimization of preserved areas crossing and equal weights.
\end{abstract}

Keywords: forest harvesting, LiDAR, precision management.

\section{INTRODUÇÃO}

Um dos grandes desafios do manejo de florestas nativas é buscar um planejamento economicamente viável, que atenda de forma ótima as restrições operacionais e ambientais. Na maioria dos casos, a exploração madeireira na Amazônia é realizada em locais remotos, com escassas informações topográficas, o que implica em uma gama de questões a serem solucionadas durante o planejamento (BRAZ; D'OLIVEIRA, 2001; RIBEIRO et al. 2002). Dentre os fatores que influenciam o planejamento de uma exploração madeireira destacam-se: distribuição espacial das árvores, densidade da vegetação, rede hidrográfica, regiões de baixa drenagem e regiões de forte declividade (FIGUEIREDO et al. 2008).

\footnotetext{
${ }^{1}$ Engenhario Florestal. UFVJM - Universidade Federal dos Vales do Jequitinhonha e Mucuri. Rodovia MGT 367 - Km 583 , 5000 - 39100-000 - Diamantina.

2Professor Associado no Departamento de Ciências Florestais. USP - Universidade de São Paulo / ESALQ - Escola Superior de "Luiz Queiroz". Av. Pádua Dias, 11 - 13418900 - Piracicaba, SP, Brasil.

3Professor Associado no Departamento de Engenharia Florestal. UFVJM - Universidade Federal dos Vales do Jequitinhonha e Mucuri. Rodovia MGT 367 - Km 583, 5000 - 39100-000 - Diamantina.
}

Sci. For., Piracicaba, v. 45, n. 115, p. 541-550, set. 2017 DOI: dx.doi.org/10.18671/scifor.v45n115.11 
A determinação da melhor rota de exploração (para acesso e retirada das árvores) não deve considerar somente a minimização das distâncias, mas também a minimização do impacto ambiental e das restrições operacionais, o que torna o problema de otimização complexo e com múltiplos objetivos (BRAZ, 2002). Segundo Oliveira e Saramago (2005) a otimização multicritério permite otimizar um problema considerando múltiplos fatores simultaneamente, o que torna esta ferramenta interessante alternativa para a otimização de rotas de exploração no manejo de florestas nativas.

Dada a dificuldade em se obter informações detalhadas da área de manejo, os sensores ativos (emitem energia própria em direção aos objetos alvos para capturar a energia refletida) são uma poderosa ferramenta para a obtenção de dados do dossel e da superfície da floresta. Dentre as técnicas de sensoriamento remoto ativo, com uso promissor em áreas de manejo de florestas nativas, destaca-se o sistema laser aerotransportado (ALS) (REUTEBUCH et al., 2005).

O ALS utiliza o equipamento de transmissão e recepção laser (LiDAR - Light Detection And Ranging) acoplado ao equipamento de posicionamento global (GNSS), e à unidade de medição inercial (IMU). Toda a informação coletada é enviada e salva no microcomputador, que também é responsável pela sincronização dos dados (BALTSAVIAS, 1999).

Os múltiplos retornos dos pulsos laser permitem que diferentes camadas da estrutura vertical da paisagem sejam identificadas e mensuradas, resultando na estrutura tridimensional (REUTEBUCH et al., 2005). O levantamento laser aerotransportado é capaz de obter informações mesmo em áreas com dossel denso, devido à alta frequência do pulso laser que permite um alto nível de detalhamento das feições (RODRIGUEZ et al., 2010). A tecnologia LiDAR vem sendo utilizada com sucesso como ferramenta de quantificação de florestas devido à alta correlação entre as métricas extraídas da nuvem e os atributos florestais (GOODWIN et al., 2006).

O objetivo deste estudo foi utilizar a otimização multicritério para determinar a rota ótima para exploração madeireira a partir de dados obtidos por meio de levantamento laser aerotransportado. Formula-se a hipótese de que o alto nível de detalhamento obtido pelo sensor laser das feições da vegetação e do terreno, aliado à alta correlação das métricas LiDAR com atributos florestais, será possível considerar quatro critérios de otimização: menor distância, minimizar as intervenções em área de maior densidade de vegetação, minimizar a intervenção em áreas de preservação e minimizar a intervenção em áreas de maior declividade.

\section{MATERIAL E MÉTODOS}

O trabalho foi desenvolvido em um transecto de 500 ha na Floresta Nacional (FLONA) do Jamari, localizada ao Norte do Estado de Rondônia, entre os municípios de Candeias do Jamari, Itapuã do Oeste e Cujubim (latitude $0900^{\prime} 00^{\prime \prime}$ a $0930^{\prime} 00^{\prime \prime}$ S e longitude $6244^{\prime} 05^{\prime \prime}$ a $6316^{\prime} 54^{\prime \prime} \mathrm{W}$, EPSG 4326). O clima da região segundo Köppen é Aw-, caracterizado como clima tropical chuvoso, com precipitação entre 2.200 e 2.600 mm. $\mathrm{ano}^{-1}$ (ICMBIO, 2016).

\section{Levantamento laser aerotransportado}

O levantamento laser aerotransportado foi realizado em novembro de 2011 sobrevoando a área a uma altura média de $850 \mathrm{~m}$, com o sensor LiDAR ALTM 3100, emitindo pulsos a $100 \mathrm{kHz}$, um GNSS APLANIX 09SEN243 e o IMU LITTON 413996. O ângulo de varredura foi de $11^{\circ}$, com sobreposição de faixas de 65\%. Os demais parâmetros do levantamento se encontram detalhados na Tabela 1.

Tabela 1. Parâmetros do levantamento lase aerotransportado.

Table 1. Airborne laser scanning parameters.

\begin{tabular}{lc}
\hline Parâmetro & Valor \\
\hline Data de voo & $20 / 11 / 2011$ \\
Datum & WGS84 \\
Zona UTM & $20 \mathrm{~S}$ \\
Área sobrevoada & $500 \mathrm{ha}$ \\
Densidade média de retornos & $25,8 \mathrm{pts} . \mathrm{m}^{-2}$ \\
Densidade média de primeiro retorno & $16,15 \mathrm{pts} \cdot \mathrm{m}^{-2}$ \\
\hline
\end{tabular}

\section{Modelo digital de terreno e modelo digital de altura de dossel}


O modelo digital de terreno (MDT) foi criado com resolução espacial de 1 metro, a partir do valor médio dos retornos classificados como solo (Figura 1). Para a classificação dos retornos de solo, foi utilizado o filtro de Kraus e Pfeifer (1998), com janela fixa de 8 metros e parâmetros da função de ponderação dos pesos de: $\mathrm{g}=-2 ; \mathrm{w}=2,5 ; \mathrm{a}=1 \mathrm{e} \mathrm{b}=4$. O modelo digital de altura de dossel (MDD) foi gerado a partir do retorno de máxima altura dentro de cada pixel de 1x1 m, já normalizado pelo modelo digital de terreno (Figura 2).

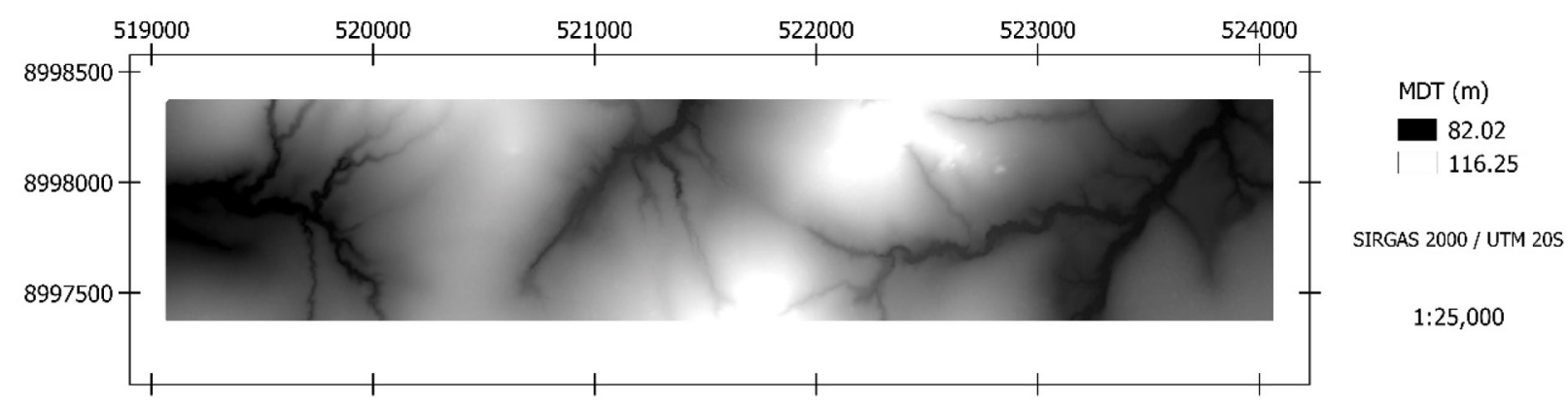

Figura 1. Modelo digital de terreno da área de estudo.

Figure 1. Digital terrain model for the study area.

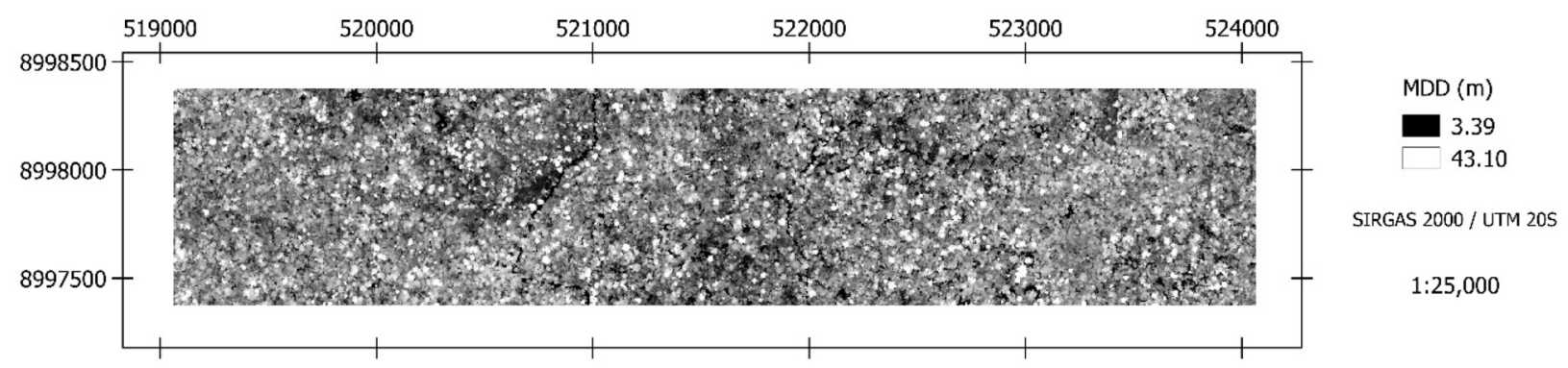

Figura 2. Modelo digital de altura de dossel da área de estudo.

Figure 2. Canopy height profile for the study area.

\section{Critérios de otimização}

Foram derivados da nuvem LiDAR quatro critérios a serem considerados na otimização: distância inclinada (considera a variação horizontal e vertical), declividade, altura da vegetação e localização das áreas de preservação.

A distância inclinada é o critério utilizado como referência durante qualquer problema de roteamento. Com base no modelo digital de terreno, a distância é obtida a partir do deslocamento horizontal, levando-se em consideração a variação vertical, resultando no que se conhece por distância inclinada.

O modelo digital de inclinação (MDI) foi gerado atribuindo a cada pixel o maior gradiente de variação da elevação em relação aos seus vizinhos (Figura 3). Em seguida, os valores de inclinação foram classificados, considerando a versão adaptada da classificação da inclinação e formas de relevo da EMBRAPA (2006). Cada classe foi associada à uma penalidade: 5 para declividades superiores a $8 \%, 3$ para declividades entre 3 e $8 \%$ e 1 para declividades inferiores a 3\% (Tabela 2).

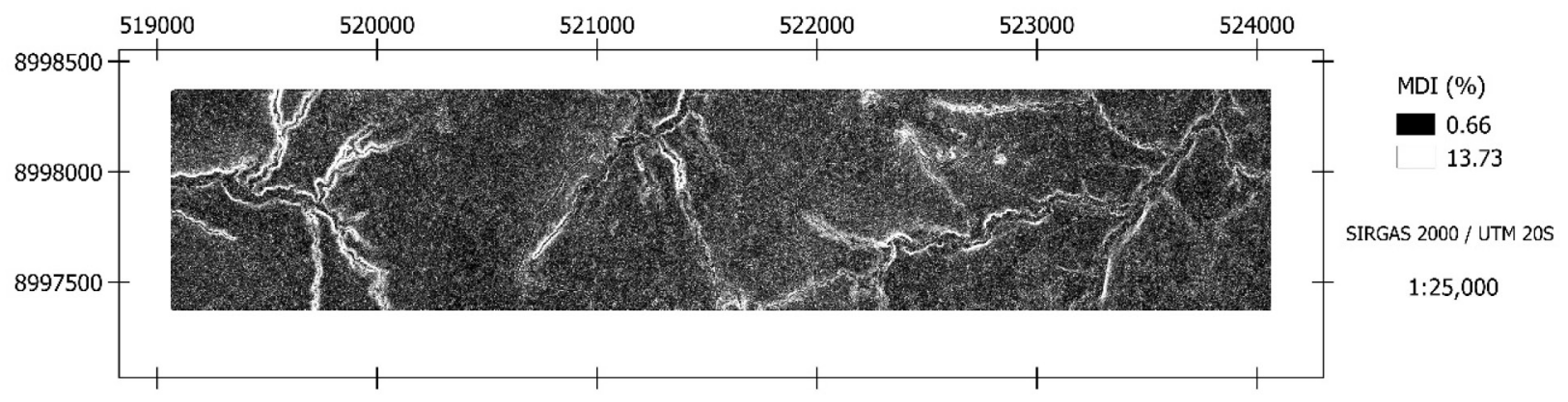

Figura 3. Modelo digital de inclinação da área de estudo.

Figure 3. Digital slope model for the study area. 
Tabela 2. Classificação da declividade da área e o respectivo coeficiente de penalização para a criação da superfície de custo.

Table 2. Classification of the slope and the respective penalty for the cost surface creation.

\begin{tabular}{lcc}
\hline Inclinação \% & Classe & Coeficiente \\
\hline $0-3$ & Muito baixo & 1 \\
$3-8$ & Baixo & 3 \\
$>8$ & Médio a muito alto & 5 \\
\hline
\end{tabular}

O porte da vegetação foi modelado pelo valor de altura da vegetação determinado pelo modelo digital de altura de dossel (MDA). Visando minimizar a intervenção em áreas com vegetação de maior porte, o MDA foi classificado em três classes de altura e uma penalização foi atribuída a cada classe: a penalidade da vegetação com altura maior que $20 \mathrm{~m}$ foi de 5, para vegetação entre 11 a 20 $\mathrm{m}$ foi de 3 e para a vegetação com altura inferior a $11 \mathrm{~m}$ foi de 1 (Tabela 3 ).

Tabela 3. Classificação do modelo digital de altura de dossel e o respectivo coeficiente de penalização para a criação da superfície de custo.

Table 3. Classification of canopy height model and respective penalty for the cost surface creation.

\begin{tabular}{lcc}
\hline Altura $(\mathbf{m})$ & Classe & Coeficiente \\
\hline Menor do que 11 & Muito baixo & 1 \\
$11-20$ & Baixo & 3 \\
Maior do que 20 & Médio a muito alto & 5 \\
\hline
\end{tabular}

O quarto critério considerado durante a determinação da rota ótima foi à localização das Áreas de Preservação Permanente (APP). Segundo o Código Florestal (Lei 12.651 de 2012), a APP é uma área protegida, coberta ou não por vegetação nativa, com a função ambiental de preservar dentre outros, os recursos hídricos. Este trabalho considerou apenas as APP na margem de rios, e as mesmas foram consideradas como penalidades e não como restrições, uma vez que restringir completamente o acesso faria com que as áreas ficassem inacessíveis devido às características da rede hidrográfica da área de estudo. Na prática, este problema seria contornado por meio de vizinhos ou por meio de ramais já existentes. No entanto, não há levantamento laser disponível sobre as áreas vizinhas para incluirmos eventuais desvios neste estudo.

A determinação das APP's partiu do modelo hidrográfico da área, extraído do MDT. O primeiro passo foi corrigir o MDT, suavizando pequenas imperfeições e preenchendo por meio de interpolação com os vizinhos mais próximos as lacunas do modelo digital de terreno. A partir do MDT corrigido, a rede hidrográfica foi obtida primeiro determinando a superfície de direção de fluxo e em seguida, a superfície de acumulação de fluxo. A direção de fluxo de cada pixel foi identificada comparando com a elevação dos respectivos oito vizinhos diretos (numa estrutura de 3 pixels por 3 pixels, formando uma região quadrangular de nove pixels, o pixel central possui 8 vizinhos). Em seguida, a superfície de acumulação de fluxo foi criada de acordo com a direção de fluxo. De posse das superfícies de direção e acumulação de fluxo obteve-se a rede hidrográfica a partir de um limiar de acumulação. A partir da malha hídrica determinada, a APP pode ser automaticamente delimitada utilizando a análise de proximidade, considerando uma largura fixa de 30 metros (largura mínima exigida pelo código florestal para cursos d'água com largura inferior a 10 metros) (figura 4). A presença de APP resultou na penalidade de 5, e a ausência em penalidade igual a 1.

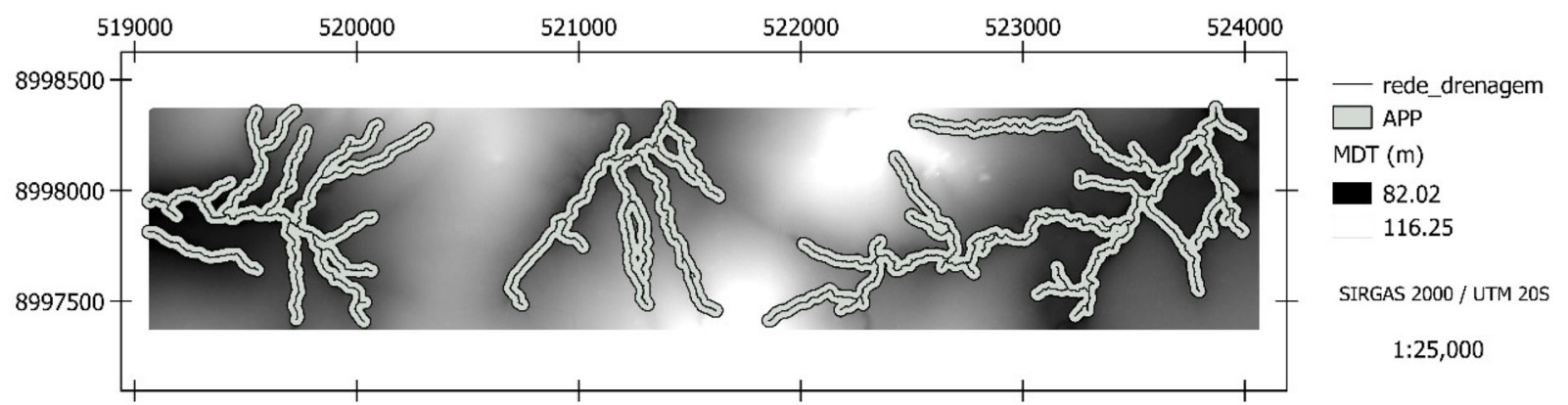

Figura 4. Linhas de drenagem extraídas com base no modelo digital de terreno e delimitações das Áreas de Preservação Permanentes (APP's).

Figure 4. The water flow network extracted from the digital terrain model and permanent preservation area defined as 30 metros from both sides of the water stream. 


\section{Pontos de interesse (origem e destinos)}

Neste estudo, foi definido de forma arbitrária um ponto na borda sul da área denominado de ponto de extração (ou ponto de origem). Foram lançadas de modo aleatório dentro da área de estudo (desconsiderando as APP's) 63 árvores, que para o modelo serão consideradas como pontos de destino. Como resultado, o problema toma forma como roteirização entre um ponto de origem e múltiplos pontos de destino (Figura 5).

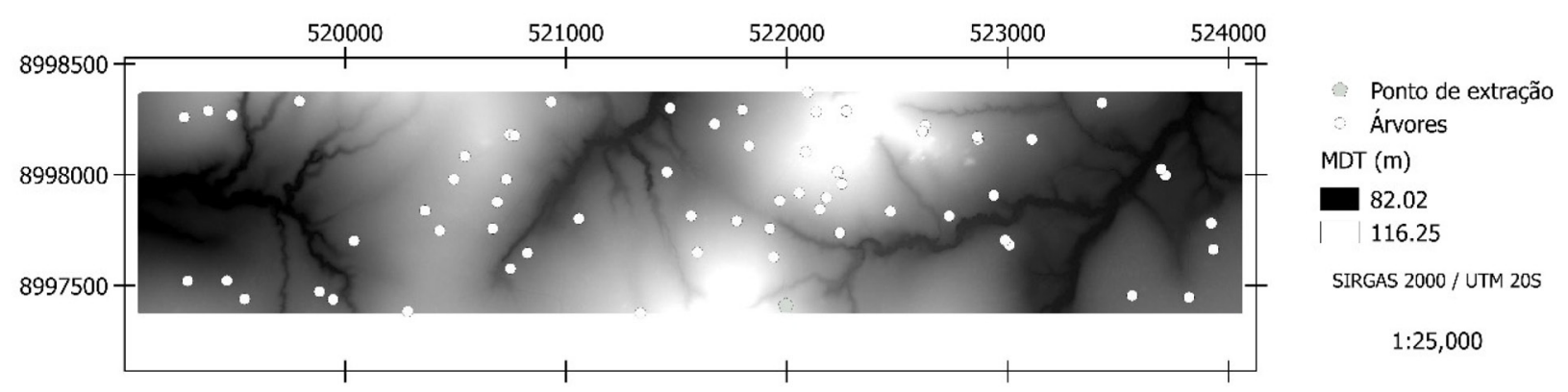

Figura 5. Modelo digital de terreno da área de estudo, com a localização das árvores (círculo) com o ponto de extração (pentágono).

Figure 5. Digital terrain model for the study area and the position of trees (circles) and extract point (pentagon).

\section{Superfícies de custo e de distância ponderada}

Esta superfície de custo é obtida pela soma das penalidades definidas para cada classe do respectivo critério, ponderado pelo grau de importância daquele critério.

Analisaram-se quatro cenários para a construção da superfície de custo, variando a importância de cada critério conforme definido na Tabela 4. Um cenário considerou com igual importância todos os critérios. Num segundo cenário priorizou-se a minimização dos impactos na vegetação de maior porte. Em outro cenário priorizou-se a minimização de rampas ao longo do trajeto. No quarto cenário, minimiza-se o impacto sobre as Áreas de Preservação Permanentes.

Tabela 4. Grau de importância dos critérios nos diferentes cenários analisados.

Table 4. Importance weight for each criterion in each analyzed scenario.

\begin{tabular}{lccc}
\hline Cenário & MDA & MDI & APP \\
\hline Minimização balanceada & 33,3 & 33,3 & 33,3 \\
Minimização do impacto à vegetação & 80 & 10 & 10 \\
Minimização de rampas & 10 & 80 & 10 \\
Minimização de impacto à APP & 10 & 10 & 80 \\
\hline
\end{tabular}

A superfície de distância ponderada determina a menor distância ponderada pela superfície de custo entre cada pixel da área de estudo e o local de origem (ponto de extração). A distância ponderada de cada pixel é o resultado da multiplicação da menor distância inclinada do ponto de origem até o pixel pelo valor do pixel na superfície de custo considerando os outros critérios de otimização (altura da vegetação, declividade e existência de APP).

\section{Otimização}

Por fim, estimou-se o caminho de menor custo da origem até cada um dos destinos (árvores), buscando delinear o caminho que passa pelos pixels de menor custo acumulado considerando cada um dos cenários de otimização. Os cenários foram comparados considerando a distância total percorrida e o número de interceptações à APP. Vale destacar que num processo de otimização, o resultado obtido é ótimo e as diferenças absolutas entre os cenários são por si só significativas. Numa situação real, portanto, cabe ao analista compreender o custo associado ao desvio da solução ótima, determinando se o desvio é aceitável ou não.

\section{RESULTADOS E DISCUSSÃO}

Todos os cenários apresentaram um caminho ótimo para deslocamento entre o ponto de extração (origem) e as árvores (múltiplos pontos de destino). A superfície de custo do cenário de mini- 
mização de impacto à APP (Figura 6) mostra a imagem saturada (escura), resultado de uma grande quantidade de pixels classificados como de baixo custo. Ao atribuir peso maior a um fator binário (presença ou ausência de APP), este cenário atribui importância muito baixa aos demais fatores.

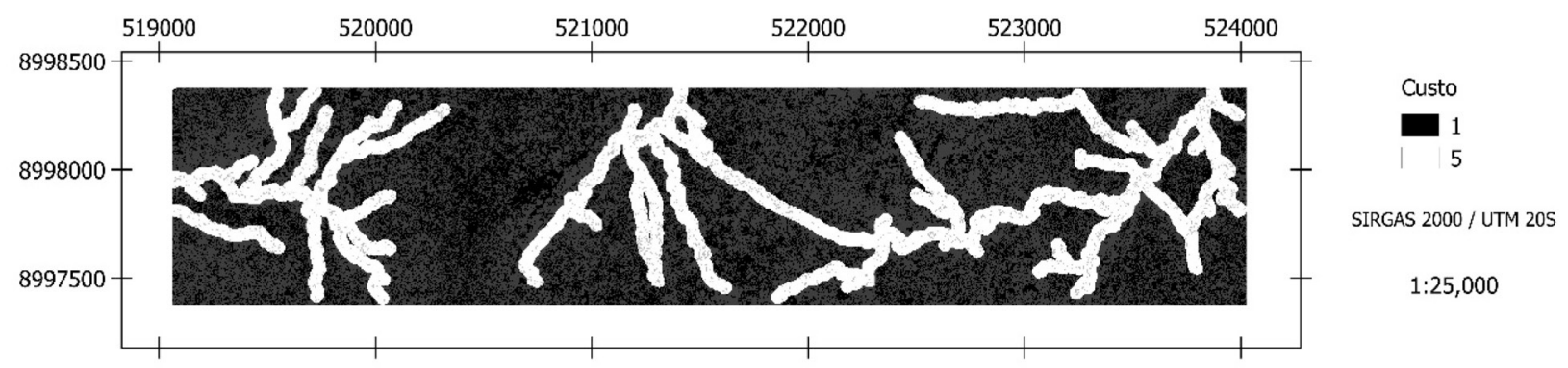

Figura 6. Superfície de custo do cenário de minimização de impacto à APP.

Figure 6. Cost surface for minimizing the impact over permanent preservation areas scenario.

O cenário de minimização de impacto sobre a vegetação classifica grande parte dos pixels como alto custo, resultando também em uma imagem saturada (clara) (Figura 7). Por se tratar de uma floresta ombrófila, o componente vegetal possui um componente arbóreo significativo na estrutura vertical. A distribuição horizontal uniforme dos componentes arbóreos dominantes leva à superfície constante e de alto custo.

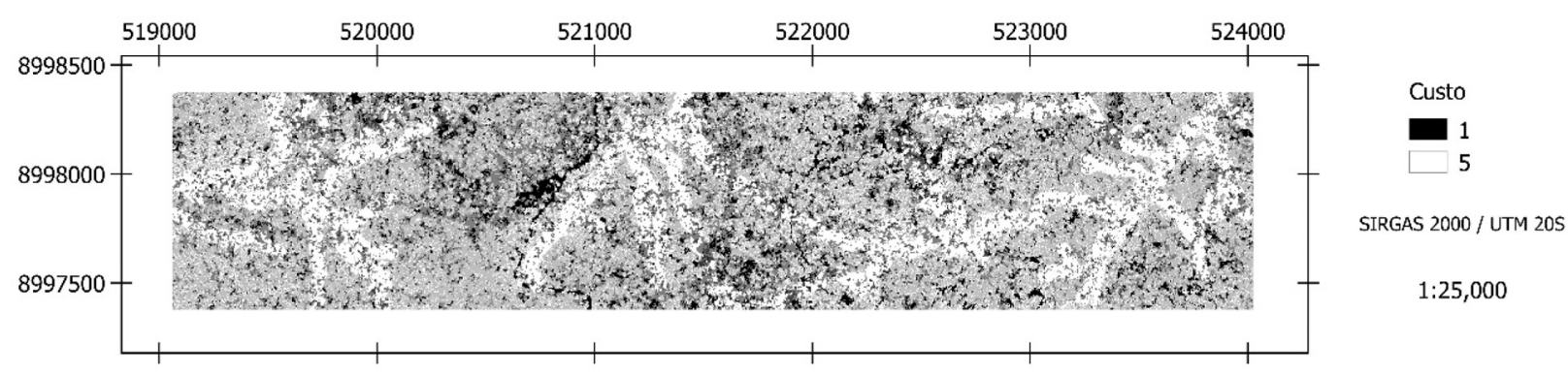

Figura 7. Superfície de custo do cenário de minimização do impacto à vegetação.

Figure 7. Cost surface for minimizing the impact over the vegetation scenario.

Tanto o cenário de minimização de rampa (Figura 8) quanto o cenário de minimização balanceada (Figura 9) apresentaram superfícies de custo mais equilibradas, devido à melhor combinação dos critérios, bem como, numa melhor distribuição espacial dos componentes do custo ao longo da área de estudo.

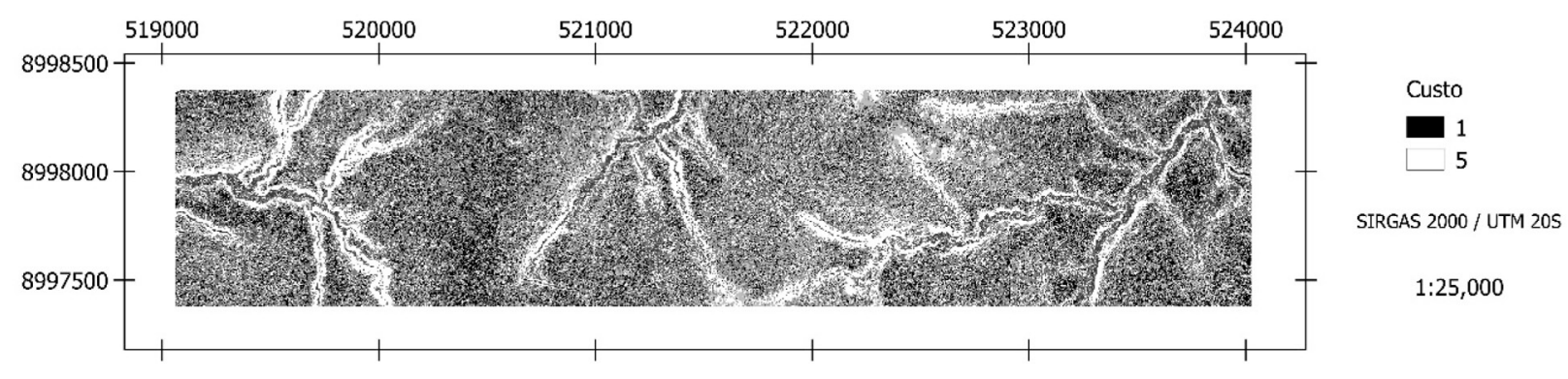

Figura 8. Superfície de custo do cenário de minimização de rampas ao longo da rota.

Figure 8. Cost surface for minimizing the slope along tracks scenario.

No cenário de minimização em que os critérios foram considerados com igual importância, o caminhamento total resulta em 26,95 km e 30 interceptações com APPs (Figura 10). No cenário de minimização do impacto sobre a vegetação, a rota atingiu extensão total de $26,15 \mathrm{~km}$ e 42 interceptações com APPs (Figura 11). No cenário que minimiza, a extensão total da rota foi de 30,92 km e 52 interceptações à APPs (Figura 12). No quarto cenário, de minimização de interceptação de APP (Figura 13), a extensão total da rota foi de 26,31 km e 17 interceptações de APP (Tabela 5). 


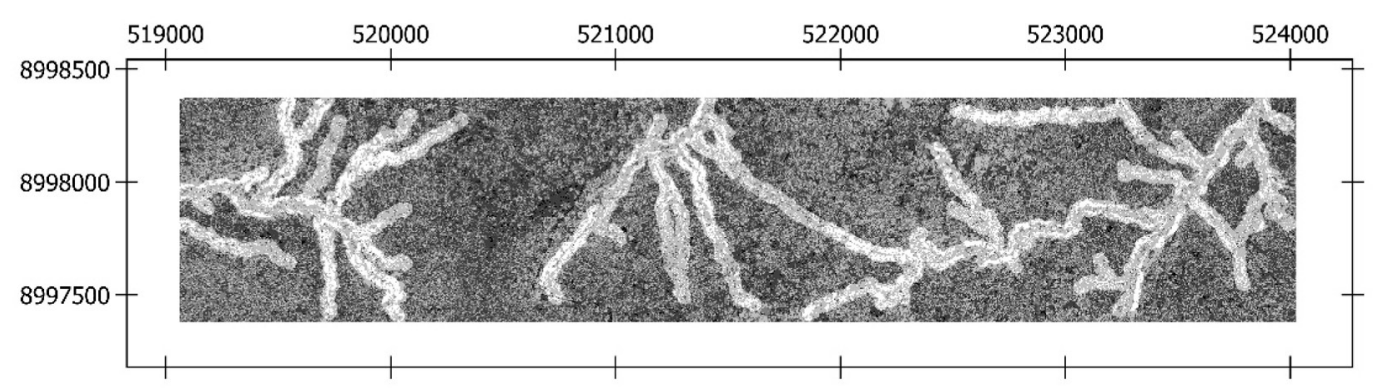

Figura 9. Superfície de custo do cenário de minimização balanceada.

Figure 9. Cost surface for minimizing the balanced criteria scenario.

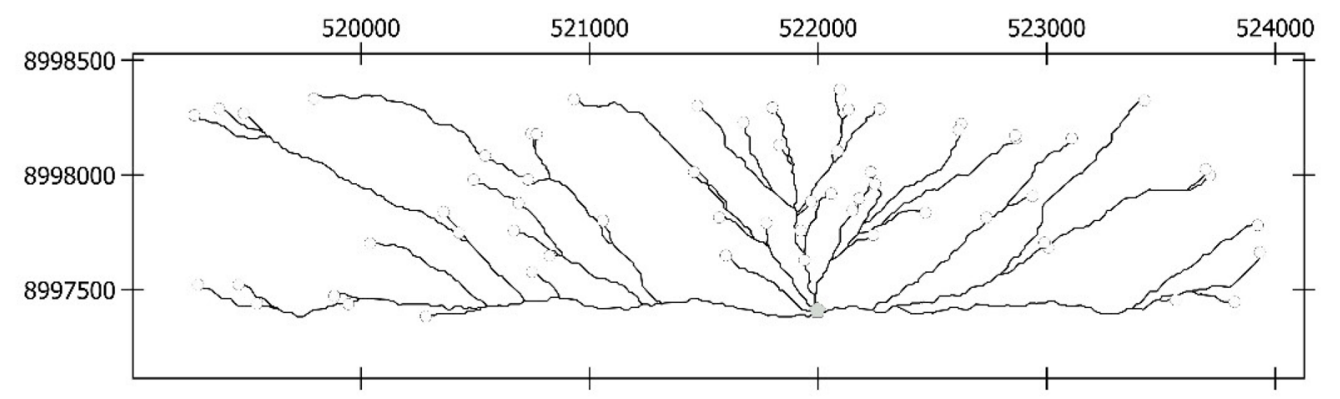

Ponto de extração Árvores

- Caminho ótimo

SIRGAS $2000 /$ UTM 20 S

$1: 25,000$

Figura 10. Rota de extração ótima para o cenário de minimização balanceada.

Figure 10. Best extraction route for the balanced criteria scenario.

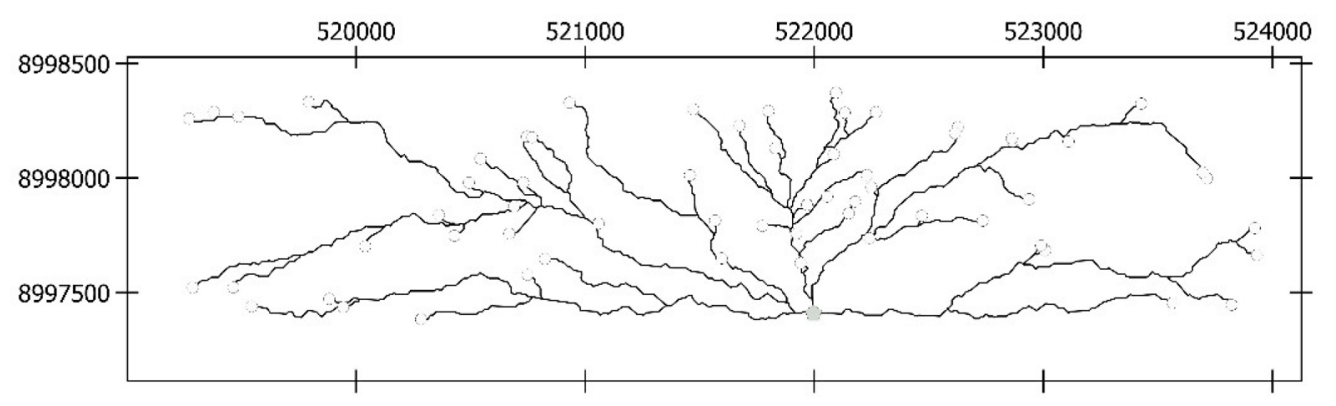

Ponto de extração Árvores

- Caminho ótimo

SIRGAS 2000 / UTM 20 S

$1: 25,000$

Figura 11. Rota de extração ótima para o cenário de minimização do impacto à vegetação. Figure 11. Best extraction route for the minimizing impact over the vegetation scenario.

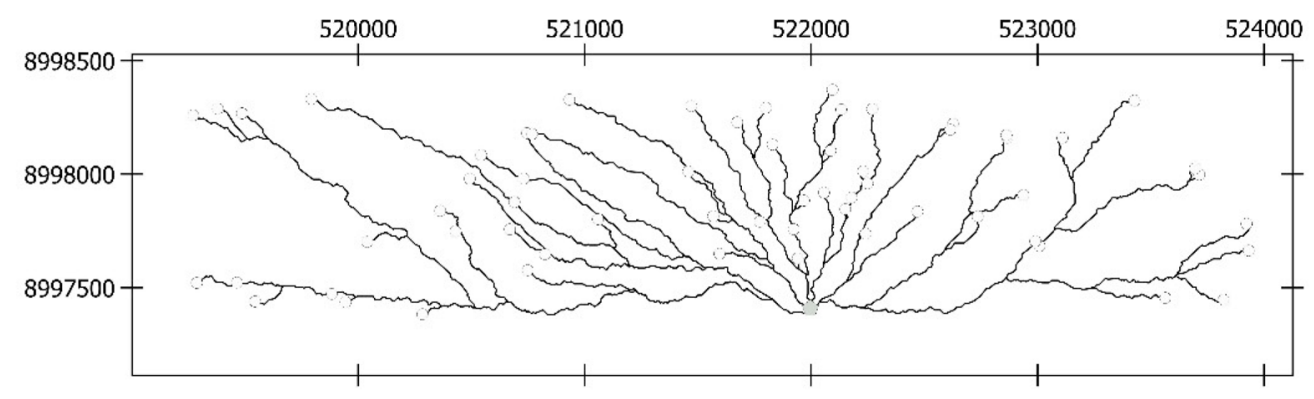

Ponto de extração Árvores

- Caminho ótimo

SIRGAS 2000 / UTM 205

$1: 25,000$

Figura 12. Rota de extração ótima para o cenário de minimização de rampas.

Figure 12. Best extraction route for the minimizing slope along tracks scenario.

Tabela 5. Extensão em quilômetros do caminho de cada cenário e número de interceptações às Áreas de Preservação Permanentes.

Table 5. Extension in kilometers of the path for each scenario and the number of interceptions to permanent preservation areas.

\begin{tabular}{lcc}
\hline Cenário & Extensão (km) & Interceptação APP \\
\hline Minimização balanceada & 26,95 & 30 \\
Minimização do impacto a vegetação & 26,15 & 42 \\
Minimização de rampas & 30,92 & 52 \\
Minimização de impacto a APP & 26,31 & 17 \\
\hline
\end{tabular}




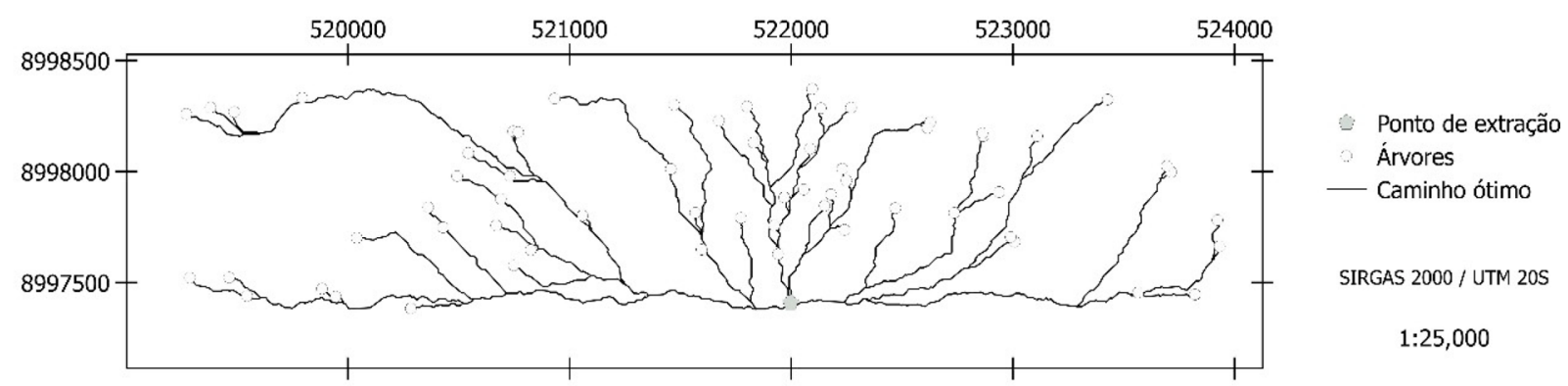

Figura 13. Rota de extração ótima para o cenário de minimização de interceptação de APP.

Figure 13. Best extraction route for minimizing the impact over permanent preservation areas.

No planejamento do manejo florestal na Amazônia, a principal ferramenta de sensoriamento remoto atualmente utilizada para realização do macrozoneamento são as imagens de satélites de média resolução e as cartas planialtimétricas do IBGE (Instituto Brasileiro de Geografia e Estatística). Como resultado, o planejamento florestal sofre com a falta de detalhamento para a correta determinação das características ambientais (topografia, hidrografia, regiões alagadas, distribuição da vegetação), trazendo grande subjetividade na etapa de microzoneamento das unidades de exploração. Em muitos casos, deixa-se de explorar determinadas áreas, sob o pretexto de serem consideradas inacessíveis, mesmo que a mesma área seja perfeitamente explorável (BRAZ, 2002).

Rogers e Schiess (2001) utilizaram a metodologia automatizada para locação de estradas florestais minimizando a distância, implementada no Arcview (extensão PEGGER) a partir de curvas de nível do terreno. Porém, no manejo de florestas nativas, deixar de considerar os fatores ambientais resulta em trilhas de arrastes inadequadas, em baixo rendimento operacional durante a construção dos ramais e em maior manutenção, além de maiores danos ambientais (BRAZ, 2002).

Neste contexto, é evidente que a obtenção de dados detalhados possibilita melhor estimação dos fatores que influenciam os custos de construção de estradas, além de permitir a melhor consideração dos impactos ambientais, uma vez que serão necessários menos obras de arte como bueiros e pontes (FIGUEIREDO et al. 2008).

Pereira et al. (2013) compararam um MDT derivado de ALS com demais fontes de dados comumente usadas no sensoriamento remoto (ex: modelos digitais de terreno derivados do SRTM). A partir de testes estatísticos e análise visual, foi possível constatar que o MDT gerado a partir do ALS possui grande vantagem frente aos demais, devido a características como: alta acurácia dos valores altimétricos; maior detalhamento da morfologia da superfície sendo possível identificar intervenções antrópicas como estradas e caminhos florestais, melhorando a definição de feições do relevo.

O cenário de minimização de rampas é interessante para antecipar restrições operacionais para os equipamentos florestais como tratores e caminhões, bem como reduzir custos associados à construção e manutenção de estradas. Lima et al. (1998) cita que para que o Skidder possa trafegar garantindo segurança operacional e ergonômica no que tange a estabilidade e dirigibilidade, as rampas de inclinação do terreno não devem ultrapassar o limite de 33,2\% de inclinação (18,37 graus). Embora tratores de arraste de toras (Skidder) sejam tolerantes às maiores inclinações, caminhões carregados suportam declividade de até 9\% (5,14 graus) desde que o leito da estrada seja bem estruturado. Embora não explorada neste trabalho, tal diferenciação pode ser útil para a definição de pátios intermediários, no qual a transposição de modais ocorreria (arraste - transporte).

Todos os cenários resultaram em interceptações na APP. Figueiredo et al.(2008) sugerem dentro do planejamento de trilhas de arraste, que as APP's sejam indicativo de pontos críticos de risco. Estes locais são zonas em que o solo possui resistência ao rolamento, podendo subtrair a tração do trator florestal (BRAZ et al., 2001), além de apresentar maior acúmulo hídrico, o que implica em maior manutenção e necessidade de construções de pontes e bueiros, elevando os custos de implantação. Devido às limitações da extensão da área coberta com informações do sensoriamento remoto laser, é notório que a intercepção de APP seja inevitável. Outro ponto que merece destaque é que este estudo parte da premissa que não existe rede viária na área. Em um caso real, eventuais malhas existentes seriam aproveitadas, bem como áreas vizinhas poderiam ser incluídas como rotas alternativas para extração. 
Os resultados indicam que os cenários mais adequados são: minimização de impacto à APP e minimização balanceada, com 17 e 30 interceptações, respectivamente, nas APP's. Além da questão legal relacionada à intervenção em APP, a menor interceptação implica em menores custos de manutenção de estradas, devido à menor necessidade de obras de infraestrutura uma vez que estas zonas geralmente correspondem a locais de acúmulo de fluxo, baixa drenagem e rampas com fortes aclives e declives (FIGUEIREDO et al. 2008).

\section{CONCLUSÕES}

A otimização multicritério se mostrou de grande valia ao permitir combinar diferentes fatores na composição da superfície de custo que será otimizada. Diferentes combinações podem ser adotadas visando priorizar um ou um conjunto de fatores, resultando em diferenças entre os resultados obtidos na otimização.

A crescente disponibilidade de sensores tem aumentado de forma considerável a quantidade e o detalhamento das informações disponíveis sobre a área de manejo. Como resultado, a complexidade envolvida na tomada de decisão também aumenta, e ferramentas de suporte baseadas em métodos de otimização são cada vez mais essenciais.

Como sugestão de trabalhos futuros, recomenda-se a validação desta metodologia utilizando a localização de árvores reais, bem como incorporar os eventuais ramais existentes.

\section{AGRADECIMENTOS}

Agradecemos ao programa Paisagens Sustentáveis por disponibilizar o conjunto dados do levantamento laser aerotransportado. Agradecemos aos laboratórios de Geoprocessamento e ao Treelab, ambos da UFVJM.

\section{REFERÊNCIAS BIBLIOGRÁFICAS}

BALTSAVIAS, E. P. Airborne laser scanning: existing systems and firms and other resources. ISPRS Journal of Photogrammetry and Remote Sensing, v. 54, n. 2, p. 164-198, 1999.

BRAZ, E. M. Manejo da floresta nativa e sua viabilidade. In: CONGRESSO IBERO-AMERICANO DE PESQUISA E DESENVOLVIMENTO DE PRODUTOS FLORESTAIS. 2. Curitiba, 2002. Anais... Curitiba: FUPEF, 2002. 11 p.

BRAZ, E, M; D'OLIVEIRA, M. V. N. Planejamento da extração madeireira dentro de critérios econômicos e ambientais. Rio Branco: Embrapa Acre, 2001. 16 p.

EMBRAPA - EMPRESA BRASILEIRA DE PESQUISA AGROPECUÁRIA. Sistema brasileiro de classificação de solos. Rio de Janeiro: Embrapa Solos, 412 p. 2006.

FIGUEIREDO, E. O.; BRAZ, E. M.; D'OLIVEIRA, M. V. N. Manejo de precisão em florestas tropicais: Modelo Digital de Exploração Florestal. Rio Branco: Embrapa Acre, 2008. 9 p.

GOODWIN, N. R.; COOPS, N.C.; CULVENOR, D.S. Assessment of forest structure with airborne LiDAR and the effects of platform altitude. Remote Sensing of Environment, New York, v. 103, n. 2, p. 140-152, 2006.

ICMBIO. Plano de Manejo da • Floresta Nacional do Jamari. 2016. Disponível em: < www.icmbio.gov.br/.. planos-de-manejo/flona jamari pm diagnostico.pdf >. Acesso em: 10 de jan. 2016.

KRAUS, K.; PFEIFER, N. Determination of terrain models in wooded areas with Airborne laser scanner data. ISPRS Jornal of Photogrammetry and Remote Sensing, Amsterdam, v. 53, n. 4, p. 193-203, 1998. 
Barbosa et al. - Otimização multicritério da extração madeireira

na Amazônia com o uso de escaneamento laser aerotransportado

LIMA, J. S. S. Avaliação da força de arraste, compactação do solo e fatores ergonômicos num sistema de colheita de madeira utilizando os tratores "Feller-buncher" e "Skidder". 1998. 128 p. Tese (Doutorado em Ciência Florestal) - Universidade Federal de Viçosa, Viçosa, MG.

OLIVEIRA, L. S.; SARAMAGO, S. F. P. Seleção de geradores usando otimização multi-objetivo. 15. 2005. Uberlândia. Anais... Uberlândia: FEMEC, 2005. 11 p.

PEREIRA, A.; GOMES, A. \& PEREZ-ALBERTI, A. Vantagens da utilização de dados ALS (LiDAR) na caracterização morfológica das vertentes costeiras da Galiza Espanha): aplicação ao setor de Mougás. Revista de Geografia e Ordenamento do Território, Porto, v. 1, n. 4, p. 223-248, 2013.

REUTEBUCH, S. E.; ANDERSEN, H. E.; MCGAUGHEY, R. J. Light detection and ranging (LIDAR): an emerging tool for multiple resource inventory. Journal of Forestry, Bethesda, v. 103, n. 6, p. 286-292, 2005.

RIBEIRO, C. A. A. S. Floresta de precisão. In: MACHADO, C. C. Colheita Florestal. Viçosa: UFV, 2002.

RODRIGUEZ, L. C. E.; POLIZEL, J. L.; FERRAZ, S. F. B.; ZONETE, M. F. \& FERREIRA, M. Z. Inventário florestal com tecnologia laser aerotransportada de plantios de Eucalyptus spp no Brasil. Ambiência, Guarapuava, v. 6, p. $67-75,2010$.

ROGERS, L.; SCHIESS, P. PEGGER \& ROADVIEW. A new GIS tool to assist engineers in operations planning. Seattle: University of Washington, 2001.

Recebido em 02/08/2016

Aceito para publicação em 11/05/2017 